\title{
Effective Switch From Twice-Daily Pre-Mixed Insulin 50/50 to Liraglutide to Achieve a Good Glycemic Control in Type 2 Diabetes
}

\author{
Hidekatsu Yanaia, c, Taro Yoshimi ${ }^{\mathrm{a}}$, Ritsuko Honda ${ }^{\mathrm{b}}$
}

\begin{abstract}
The glucagon-like peptide 1 (GLP-1) stimulates insulin secretion from pancreatic $\beta$-cells in a glucose-dependent manner. Therapeutic strategies for type 2 diabetes mellitus are focused on the use of GLP-1 analogues. Liraglutide, an acylated analogue of human GLP-1, has been introduced in Japan in 2010. We will show a 70 -year-old woman with type 2 diabetes mellitus achieving a good glycemic control by switching from twice-daily pre-mixed insulin $50 / 50$ to liraglutide. Measurements of plasma glucose and serum C-peptide levels before and after each meal ingestion and daily urinary C-peptide levels suggested that liraglutide ameliorates glucose metabolism mainly not by the glucose-dependent insulinotropic effect but by amelioration in insulin sensitivity. Furthermore, results from a glucagon-loading test indicated that liraglutide reduced plasma glucose levels not by enhancing insulin secretion but by suppressing glucagon-mediated hepatic glucose output.
\end{abstract}

Keywords: C-peptide; Glucagon-like peptide-1; Glucagon-loading test; Obesity; Pre-mixed insulin

\section{Introduction}

Recently, there has been an increased attention and appreciation of the role of gastrointestinal hormones for glucose metabolism. The glucagon-like peptide 1 (GLP-1) is one of

Manuscript accepted for publication February 4, 2011

\footnotetext{
${ }^{\mathrm{a}}$ Department of Internal Medicine, National Center for Global Health and Medicine, Kohnodai Hospital, Chiba 272-8516, Japan

${ }^{\mathrm{b}}$ Department of Diabetes and Metabolic Medicine, National Center for Global Health and Medicine, Tokyo 162-8655, Japan

${ }^{\mathrm{c} C}$ Corresponding author: Hidekatsu Yanai, Department of Internal

Medicine, National Center for Global Health and Medicine, Kohnodai Hospital, 1-7-1 Kohnodai, Chiba 272-8516, Japan.

Email: dyanai@hospk.ncgm.go.jp
}

such gastrointestinal hormones. GLP-1 is produced mainly by L-cells in the ileum. GLP-1 is released into the blood from intestinal L-cells in response to meal ingestion, and GLP-1 stimulates insulin secretion from pancreatic $\beta$-cells in a glucose-dependent manner [1]. GLP-1 slows gastric emptying, which may aid weight loss, and inhibits glucagon release from the pancreatic $\alpha$-cells [1], improving insulin sensitivity.

Therefore, therapeutic strategies for type 2 diabetes mellitus are focused on the use of GLP-1 analogues. Exenatide was introduced to the market in the United States in 2005 and in Europe in 2007. Liraglutide has been introduced to the market in Europe in 2009 and in the United States and Japan in 2010 [2]. Liraglutide is an acylated analogue of human GLP-1 and has 97\% sequence homology to human GLP-1, while exenatide shares 53\% homology with human native GLP-1 [1]. Since GLP-1 is promptly inactivated by the enzyme, the dipeptidyl peptidase-4 (DPP-4), the half-life of GLP-1 is less than two minutes. A high degree of plasma protein binding capacity of liraglutide induces decreased susceptibility to metabolism by DPP-4 and the half-life of liraglutide is approximately 13 hours [3].

This article describes a 70-year-old woman with type 2 diabetes mellitus achieving a good glycemic control by switching from pre-mixed insulin 50/50 $(50 \% \mathrm{NPH}$ and $50 \%$ regular insulin) to liraglutide. Here, we will show the changes in blood glucose levels, plasma glucose and serum C-peptide levels before and after meal ingestion, results of glucagon loading test, and dairy urinary C-peptide levels, by switching from pre-mixed insulin to liraglutide.

\section{Case Report}

A 70-year-old woman, who had been diagnosed as type 2 diabetes mellitus 15 years ago, and had been treated by premixed insulin for ten years. For the last 6 months, she used pre-mixed insulin 50/50 (12 unites before breakfast and 6 unites before dinner). She had also been diagnosed as having hypertension and previous cerebral infarction, and took nifedipine ( $60 \mathrm{mg} /$ day), telmisartan ( $40 \mathrm{mg} /$ day), furosemide (40 $\mathrm{mg}$ /day), clopidogrel sulfate $(75 \mathrm{mg} /$ day), and sarpogrelate hydrochloride (300 mg/day). She did not take any oral anti- 
a

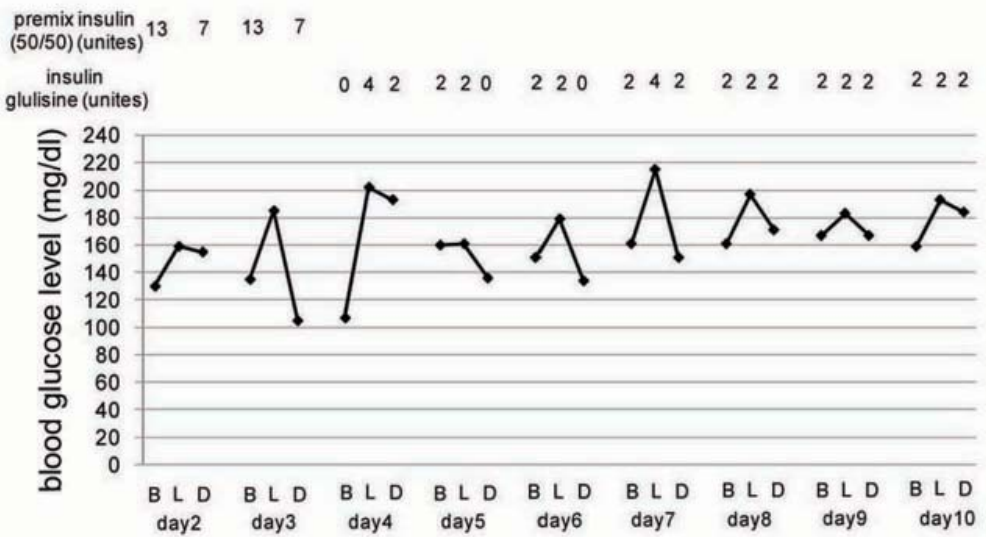

b

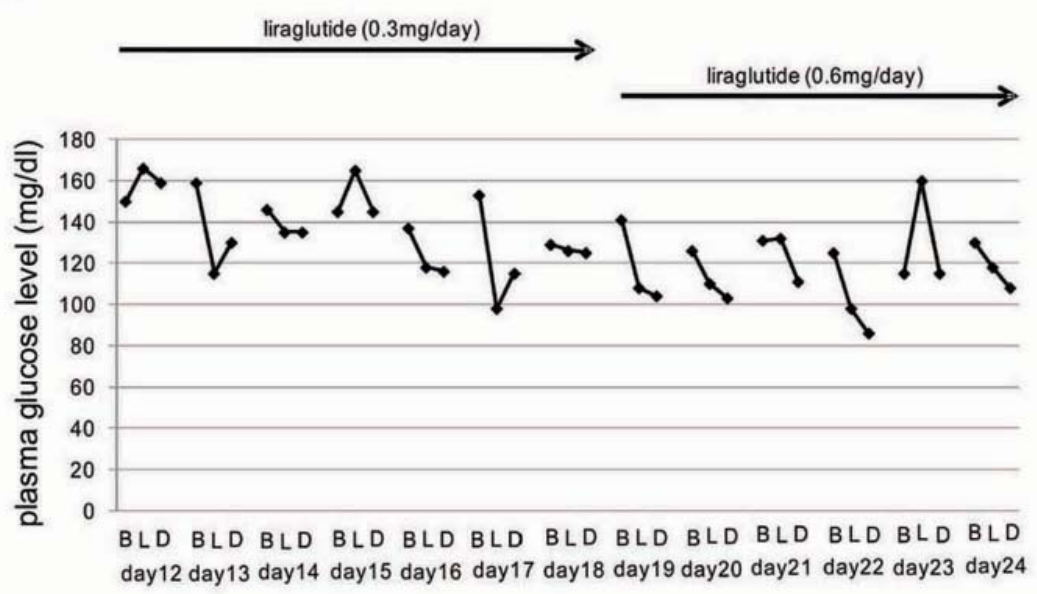

Figure 1. (a) Blood glucose levels during insulin use. (b) Blood glucose levels during liraglutide use. $B, L$ and $D$ mean before breakfast, before lunch, and before dinner, respectively. Day means the day after the admission.

diabetic drugs. Her body weight had been increasing, and her body height, body weight, and body mass index were 158 $\mathrm{cm}, 79 \mathrm{~kg}$, and $31.6 \mathrm{~kg} / \mathrm{m}^{2}$. A good glycemic control could not be obtained, and hemoglobin A1c level was $8.0 \%$ before the admission.

At first, we used pre-mixed insulin 50/50 (13 unites before breakfast and 7 unites before dinner), however, her blood glucose levels were not ameliorated. And then, we switched from twice-daily pre-mixed insulin 50/50 to threetimes-daily rapid-acting insulin glulisine. Three-times-daily low dose of rapid-acting insulin glulisine also did not ameliorate her blood glucose levels (Fig. 1a). At 11 days after the admission, we did not use insulin to washout effects of insulin. We started to use liraglutide from day 12 (12 days after the admission). Liraglutide promptly decreased blood glucose levels, and blood glucose levels with more than 150 $\mathrm{mg} / \mathrm{dl}$ were not observed except for one time spot (before lunch on day 23) while daily $0.6 \mathrm{mg}$ of liraglutide was used (Fig. 1b).

Changes in plasma glucose and serum C-peptide levels before and after meal ingestion were shown in Fig. 2. Liraglutide significantly reduced plasma glucose levels before and after each meal ingestion. Interestingly, liraglutide decreased \% serum C-peptide levels compared with those without liraglutide, indicating that liraglutide reduced plasma glucose levels by ameliorating insulin sentitivity.

We performed a glucagon loading test before and after switching from insulin to liraglutide (Fig. 3). At 6 days after the start of daily $0.6 \mathrm{mg}$ liraglutide use, increases in plasma glucose and serum C-peptide levels by a glucagon loading were significantly suppressed by liraglutide. This suggests that liraglutide reduced glucagon-mediated increase in plasma glucose and also ameliorated insulin sensitivity. In spite of that liraglutide ameliorated blood glucose levels, liraglu- 
a

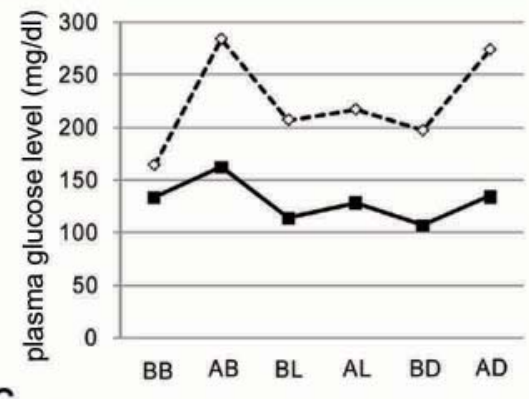

C

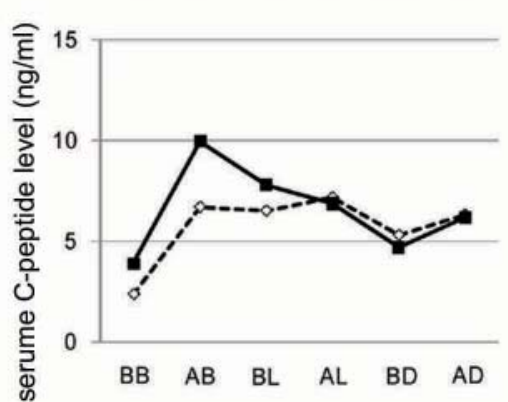

b

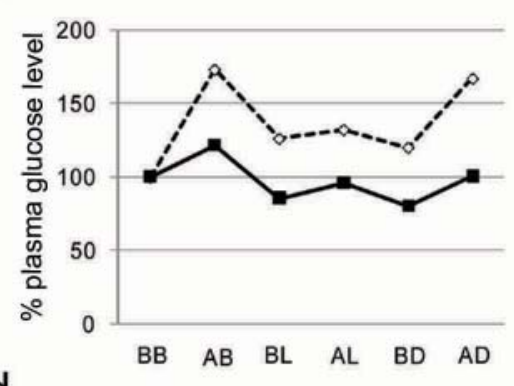

d

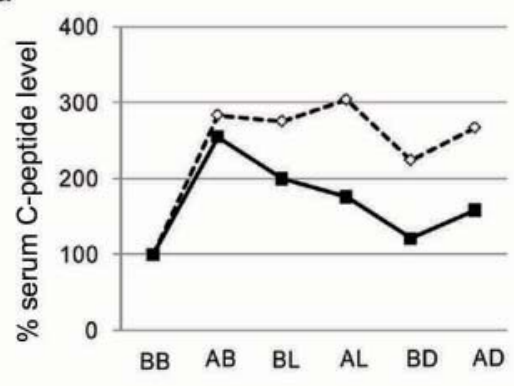

Figure 2. Plasma glucose levels (a), \% changes of plasma glucose levels from before breakfast (b), serum C-peptide levels (c), \% changes of serum C-peptide levels from before breakfast (d). Open and closed boxes indicate values before and after the liraglutide use, respectively. $B B, A B, B L, A L, B D$, and $A D$ mean before breakfast, two hours after breakfast, before lunch, two hours after lunch, before dinner, two hours after dinner, respectively.

a

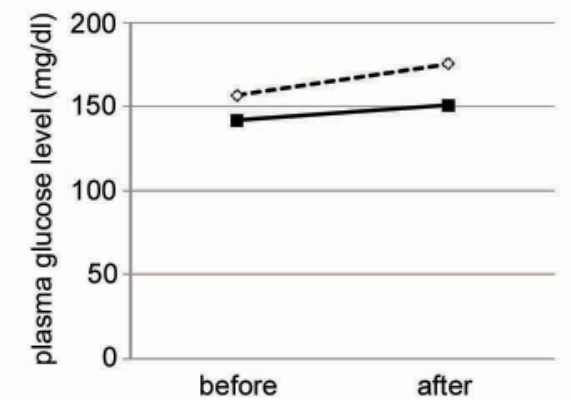

C

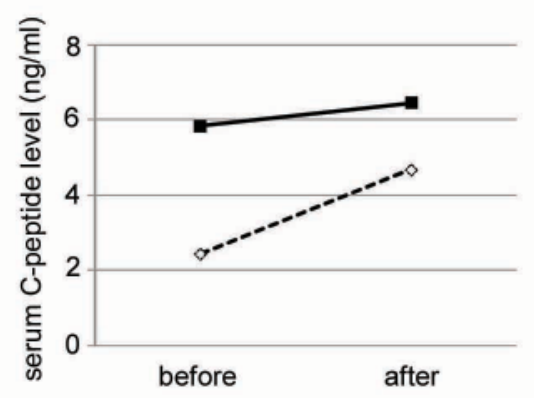

b

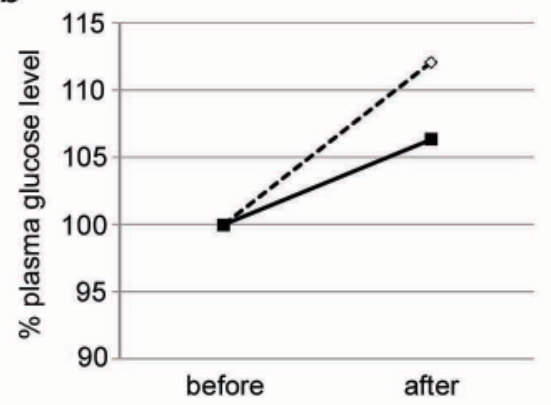

d

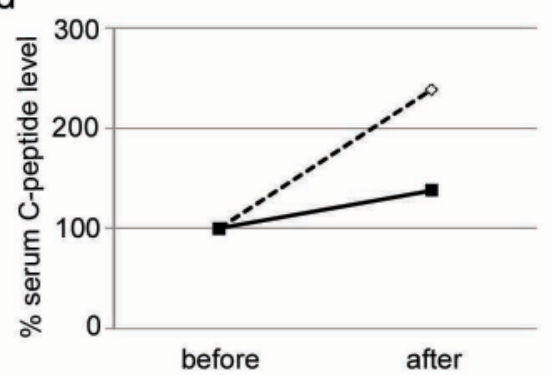

Figure 3. Changes in plasma glucose levels (a), \% changes of plasma glucose levels from values before a glucagonloading test (b), serum C-peptide levels (c), \% changes of serum C-peptide levels from values before a glucagonloading test (d). Open and closed boxes indicate values before and after the liraglutide use, respectively. 


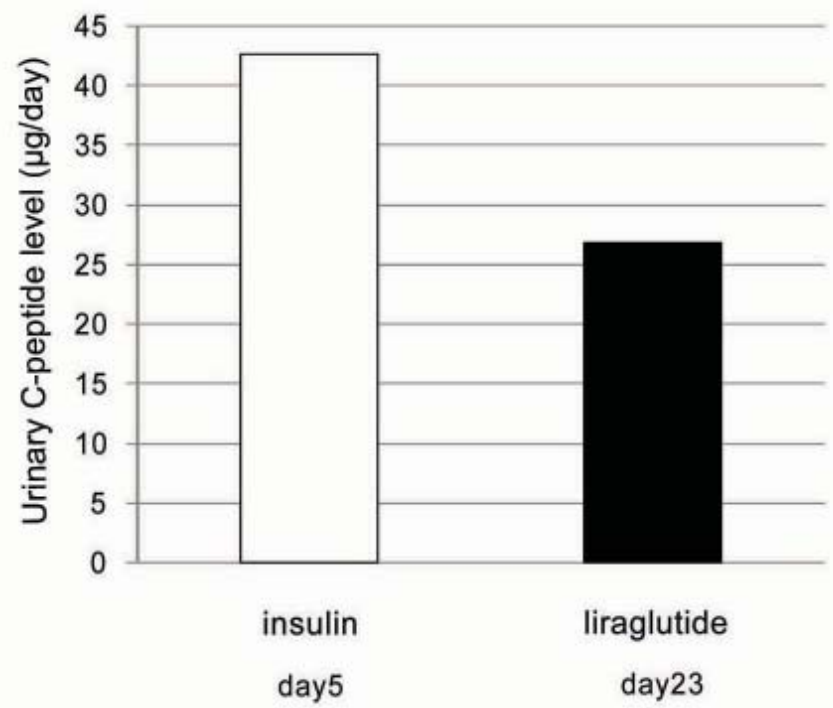

Figure 4. Daily urinary C-peptide levels during insulin use (Open box) and liraglutide use (Closed box). Day means the day after the admission.

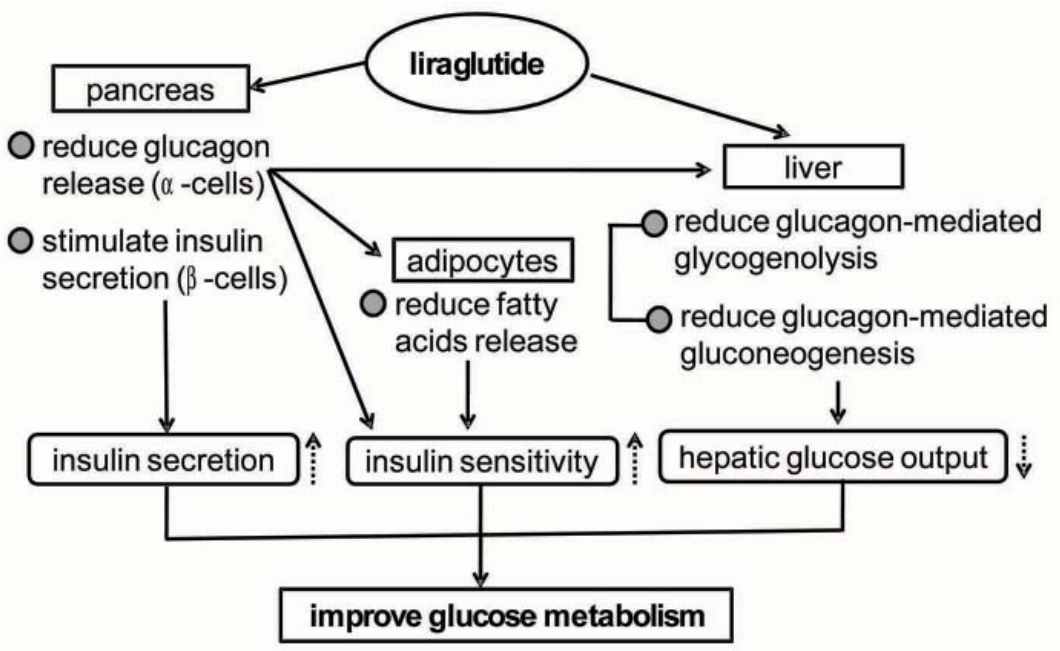

Figure 5. Possible mechanisms for the liraglutide-mediated improvements in glycemic control suggested by our study.

tide significantly reduced daily urinary C-peptide level (Fig. 4), supporting liraglutide-mediated amelioration in insulin sensitivity.

\section{Discussion}

The effects of liraglutide have been studied in the LEAD (Liraglutide Effect and Action in Diabetes). Results from the LEAD-1, 2, 3, and 5 studies indicate that liraglutide has superior (non-inferior in the LEAD-2) glycemic control compared with thiazolidinedione, sulfonylurea, and long- acting human insulin analog, and that liraglutide is likely to be more favorable for body weight reduction and the occurrence of hypoglycemia [4-7].

To our knowledge, our report is the first to observe effects of the switching from twice-daily pre-mixed insulin to liraglutide. Our patient used daily 18 unites pre-mixed insulin as monotherapy, however, a good glycemic control could not be obtained. Daily $0.6 \mathrm{mg}$ liraglutide as monotherapy significantly ameliorated glycemic control. Although liraglutide has been considered to potentiate the glucose-dependent insulinotropic and glucagonostatic effects of GLP-1, liraglutide decreased relative postprandial C-peptide levels and 
daily urinary C-peptide levels in spite of reduced postprandial plasma glucose levels in our patient. This result indicates that liraglutide decreases plasma glucose levels mainly not by the glucose-dependent insulinotropic effect but by amelioration in insulin sensitivity.

Glucagon increases plasma glucose levels by increasing hepatic glycogenolysis and gluconeogenesis from amino acids. Since glucagon has a strong insulinotropic effect, glucagon is usually used to test the potential of pancreatic $\beta$-cells to secrete insulin. In our patient, serum C-peptide level after a glucagon loading was decreased by liraglutide, in spite of reduced plasma glucose. This result suggests that liraglutide reduces glucagon-mediated increase in plasma glucose levels not by enhancing insulin secretion from pancreatic $\beta$-cell but by suppressing glucagon-mediated hepatic glycogenolysis and gluconeogenesis. Glucagon has also been reported to stimulate lipolysis in adipocytes and increase plasma free fatty acids levels [8]. Increased serum free fatty acids induce insulin resistance [9]. Suppressed lipolysis in adipocytes due to reduced serum glucagon levels by liraglutide may also be associated with amelioration in insulin sensitivity. At 2 weeks after the start of liraglutide use, she gained weight by $1.0 \mathrm{~kg}$, however, fasting and postprandial blood glucose and serum C-peptide levels were significantly reduced, indicating that liraglutide has other mechanisms to ameliorate insulin resistance than by reducing body weight. Insulin resistance may be ameliorated by an enhanced GLP-1-mediated glucagonostatic effect and the resultant suppression of lipolysis in adipocytes, and a liraglutide-mediated suppression of glucagon-mediated hepatic glucose output.

In conclusion, glycemic control in obese diabetic patients was significantly ameliorated by switching from twice-daily pre-mixed insulin (18 unites/day) to a low dose of liraglutide (0.6 mg/day). Our study suggestes various mechanisms for GLP-1 analog-mediated improvement in glycemic control (Fig. 5). However, the underlying mechanism for liraglutidemediated improvements in insulin resistance still remains obscure. Further studies, preferably with larger numbers of patients, should be performed in the future.

\section{References}

1. Kjems LL, Holst JJ, Volund A, Madsbad S. The influ- ence of GLP-1 on glucose-stimulated insulin secretion: effects on beta-cell sensitivity in type 2 and nondiabetic subjects. Diabetes 2003;52(2):380-386.

2. Hansen KB, Vilsboll T, Knop FK. Incretin mimetics: a novel therapeutic option for patients with type 2 diabetes - a review. Diabetes, Metabolic Syndrome and Obesity: Targets and Therapy 2010;3:155-163.

3. Elbrond B, Jakobsen G, Larsen S, Agerso H, Jensen LB, Rolan P, Sturis J, et al. Pharmacokinetics, pharmacodynamics, safety, and tolerability of a singledose of NN2211, a long-acting glucagon-like peptide 1 derivative, in healthy male subjects. Diabetes Care 2002;25(8):1398-1404.

4. Marre M, Shaw J, Brandle M, Bebakar WM, Kamaruddin NA, Strand J, Zdravkovic M, et al. Liraglutide, a once-daily human GLP-1 analogue, added to a sulphonylurea over 26 weeks produces greater improvements in glycaemic and weight control compared with adding rosiglitazone or placebo in subjects with Type 2 diabetes (LEAD-1 SU). Diabet Med 2009;26(3):268-278.

5. Nauck M, Frid A, Hermansen K, Shah NS, Tankova T, Mitha IH, Zdravkovic M, et al. Efficacy and safety comparison of liraglutide, glimepiride, and placebo, all in combination with metformin, in type 2 diabetes: the LEAD (liraglutide effect and action in diabetes)-2 study. Diabetes Care 2009;32(1):84-90.

6. Garber A, Henry R, Ratner R, Garcia-Hernandez PA, Rodriguez-Pattzi H, Olvera-Alvarez I, Hale PM, et al. Liraglutide versus glimepiride monotherapy for type 2 diabetes (LEAD-3 Mono): a randomised, 52-week, phase III, double-blind, parallel-treatment trial. Lancet 2009;373(9662):473-481.

7. Russell-Jones D, Vaag A, Schmitz O, Sethi BK, Lalic $\mathrm{N}$, Antic S, Zdravkovic M, et al. Liraglutide vs insulin glargine and placebo in combination with metformin and sulfonylurea therapy in type 2 diabetes mellitus (LEAD5 met+SU): a randomised controlled trial. Diabetologia 2009;52(10):2046-2055.

8. Carlson MG, Snead WL, Campbell PJ. Regulation of free fatty acid metabolism by glucagon. J Clin Endocrinol Metab 1993;77(1):11-15.

9. Chan JC, Tong PC, Critchley JA. The insulin resistance syndrome: mechanisms of clustering of cardiovascular risk. Semin Vasc Med 2002;2(1):45-57. 